Results: The time to emergence following cessation of anesthesia was $4.7 \pm 1.3$ minutes in desflurane group while it was $9.6 \pm 3.3$ minutes in propofol group $(P<0.05)$. The intraoperative hemodynamics, brain relaxation, vasomotor response and emergence characteristics were comparable in both groups. Conclusions: The use of desflurane for maintenance of anesthesia is associated with faster emergence when compared to propofol in patients undergoing resection of $\mathrm{CP}$ angle tumors. However, the intraoperative profiles were similar with the use of either anesthetic agent.

\section{Clinical outcome of intracranial aneurysms: A retrospective analysis}

\author{
Seelora Sahu, Avanish Bharadwaj, Hemant Bhagat, \\ Harsimrat Bir Singh Sodhi', Sunil Kumar Gupta' ${ }^{1}$, \\ Vinod Kumar Grover, Nidhi Bidyut Panda \\ Department of Anaesthesia and ${ }^{1}$ Neurosurgery, PGIMER, \\ Chandigarh, India
}

Background: The aim $f$ the study is to analyze the factors affecting the outcome of patients with intracranial aneurysms and to review the perioperative management of patients undergoing surgical clipping. Materials and Methods: Retrospective review of all the patients presenting for neurosurgical clipping over a period of 10 months was done. Data collected and analyzed included demographic profile, preoperative medical and surgical record, aneurysm characteristics, neurosurgical grading, anesthetic details, intra and postoperative complications and clinical outcome at discharge and six months after discharge from hospital. $P<0.05$ was considered significant. Results: Four hundred and eighty two patients of aneurysmal SAH were seen, of which 330 underwent an intervention under anesthesia; 93\% (307/330) had clipping, and 7\% (23/330) had coiling. All the factors affecting the outcome were analyzed. The patients with higher WFNS and Fischer grade, hypertension, diabetes mellitus, hydrocephalous, preoperative infarct, vasospasm, multiple aneurysms and re-bleeding were associated with poor outcome. Conclusion: From the analysis it was observed that various clinical factors and premorbid conditions were associated with a poor outcome. We conclude that the outcome in patients with aneurysmalSAH is affected by multiple factors. A thorough understanding of these factors can help us in predicting the perioperative outcome in these groups of patients.

\section{Comparison of propofol anesthesia to dexmedetomidine + propofol for intraoperative management and emergence in craniotomy surgery}

Harpreet Singh, B. L. Gupta, Meenaxi Sharma
Department of Anaesthesia, NIMS Medical College and Hospital, NIMS University, Jaipur, Rajasthan, India

Background: This study evaluated the effect of adding DEX to a total intravenous anesthetic on hemodynamic control and emergence, and requirement for antihypertensive therapy and long acting opiates. Materials and Methods: Thirty nine ASA I-III patients were enrolled into this prospective, single blind, randomized study. All patients received a standardized anesthetic and were randomized to propofol (PROP) or propofol plus DEX $(0.2-0.7 \mathrm{mcg} / \mathrm{kg} / \mathrm{hr})$ infusions during surgery. Hemodynamic data is collected continuously during the case. The emergence was videotaped and analyzed for the number of episodes and severity of coughing. Hospital length of stay was also examined. Data is analyzed using ANOVA with repeated measures and the Kruskal-Wallis test. Results: Demographic data is similar among the 2 groups. There were no adverse events or neurological outcomes in either group related to the anesthetic. Video analysis of emergence showed a tendency of increased coughing episodes for PROP vs. DEX: $5.6 \pm 8.3$ vs. $1.1 \pm 1.3(P=0.04)$ while surgeons' ratings were similar for both groups $(P=0.35)$. Mean arterial blood pressure (MAP) significantly decreased intra-operatively from baseline within groups but was not different between groups. The length of hospital stay was not significantly different for DEX and PROP groups: $2.6 \pm 1.9$, and $3.2 \pm 1.9$ days, respectively. Conclusion: DEX appears to reduce coughing therefore providing a more favorable emergence when compared to PROP. PROP + DEX is hemodynamically stable than PROP

\section{Perioperative anesthetic management and outcome of patients undergoing surgery for moyamoya disease: An institutional experience}

Navneh Samagh, Hemant Bhagat, Vinod Kumar Grover, Nidhi Panda, Sunil Kumar Gupta ${ }^{1}$

Department of Anaesthesia and ${ }^{1}$ Neurosurgery,

Postgraduate Institute of Medical Education and Research, Chandigarh, India

Background: The aim was to study the demographics, anesthetic management and outcome in patients undergoing surgery for Moyamoya disease. Materials and Methods: After obtaining institutional approval, medical records of all patients who underwent revascularization surgeries for Moyamoya disease from January 2007 to present date is included for retrospective analysis. Various preoperative, intraoperative, and postoperative data is recorded. Results: There were total of 16 patients. Six patients underwent encephaloduroarteriomyosynangiosis, two underwent 